\title{
ON ACCURACIES OF UNIAXIAL CYCLIC PLASTICITY BEHAVIOURS PREDICTED BY MULTI-SURFACE MODEL
}

\author{
By Masaru MINAGAWA*, Takeo NISHIWAKI** and Nobutoshi MASUDA***
}

\begin{abstract}
A stress-strain model which was highly accurate and distinct in nature was already proposed by the authors to estimate elasto-plastic behaviours of steel members subjected to complex repetitive loads. The model is based on one of the multi-surface plasticity theories. Material parameters introduced in the model are three functions expressing fundamental sizes of multi-surfaces and weighting functions to describe the sizes at arbitrary stress-strain phases, and one of state variables is cumulative equivalent plastic strain. Investigations of the effects of these material parameters on accuracies of stress-strain relations predicted by the model are reported.

Keywords : cyclic plasticity, uniaxial loads, multi-surface model, structural steel
\end{abstract}

\section{INTRODUCTION}

Steel sturctures are subjected at times to comparatively large loads which vary in complex ways and there is a possibility for plastic strains to occur repetitively at members or member connections. Under such conditions structural characteristics such as the load capacity or deformability will differ from those originally estimated due to the influences of loading histories. It can be considered that in extreme cases even under loads which were allowable at the time of designing, local failure or collapse of the entire structure can occur. Therefore, it is important in structural engineering to grasp elasto-plastic hysteretic characteristics of steel structures with high accuracy. In order to estimate behaviours of structures, a numerical calculation technique such as the finite element method is employed at times as an effective means. In this case, hypotheses introduced in the numerical calculations, modelling for the subject of analysis, and mathematical assumptions of material characteristics frequently have major influences on the calculated results. In case of simulating elasto-plastic hysteretic behaviours of a steel structure by numerical analysis, especially, the hysteretic characteristics of various members composing the structure must be correctly evaluated. Thus, it is an important theme to build a hysteresis model that can accurately predict elasto-plastic behaviours of steel, and a lot of constitutive models had been presented ${ }^{1) \sim 13)}$. With regard to these models, the authors discussed in a recent paper ${ }^{15)}$, and hence further discussion is omitted here.

After investigating those models, the authors proposed a model (hereafter called "the proposed model") capable of accurately expressing uniaxial quasi-static stress-strain relations ${ }^{14), 15)}$. The proposed model was

\footnotetext{
* Member of JSCE, Dr. Eng., Research Associate, Dept. of Civil Eng., Musashi Inst. of Tech.

** Member of JSCE, Dr. Eng. , Professor, Ditto

*** Member of JSCE, Dr. Eng., Associate Professor, Ditto (1-28-1 Tamazutsumi, Setagaya-ku, Tokyo 158, Japan)
} 
constructed by some modifications of the model proposed by Petersson and Popov ${ }^{10)}{ }^{11)}$ (hereafter called "Petersson-Popov Model") which had employed the multi-surface plasticity theory. In the proposed model, the method for determining material parameters necessary was clear and simple, and its effectiveness was confirmed ${ }^{14)}{ }^{15}$.

Material parameters introduced in the model are functions expressing fundamental sizes of multisurfaces and weighting functions, while state variables are cumulative equivalent plastic strain and increment of equivalent plastic strain. In this paper, emphasis is placed on finding the influences of methods for evaluating these functions and variables on prediction accuracies of uniaxial stress-strain relationships. In this context, uniaxial hysteretic stress-strain relations are calculated by means of several models including the proposed model and Petersson-Popov Model. The effectiveness of the proposed model is examined by making comparison of the calculated results and the corresponding experimental results. Firstly, outlines of Petersson-Popov Model and the model previouly built by the authors will briefly be described.

\section{PETERSSON-POPOV MODEL ${ }^{10), 11)}$}

Petersson-Popov Model expresses stress-strain behaviours by loading surfaces defined in the principal stress space. Fig. 1 gives a conceptual explanation of the correlation of uniaxial stress-strain relations and behaviours of the corresponding bi-axial multi-surfaces under uniaxial loading. A case of no pre-loading is shown in (a) and a case of being subjected to a loading history in (b). Here, for the sake of simplicity, the yield surface and loading surfaces were expediently expressed by ellipses.

In this model, the surface size function $x$, which expresses changes in the sizes of multi-surfaces at each stage of hysteresis, was expressed by the equation below.

$x=W x_{0}+(1-W) x_{\text {conv }}$

where, $\varkappa_{0}$ and $\varkappa_{c o n v}$ are functions expressing fundamental sizes of multi-surfaces (hereafter called F. S. S. functions ) and $W$ is a weighting function employed in order to describe the sizes of the surfaces at arbitrary hysteretic phases*.

$x$ is successively updated from $x_{0}$ to $x_{c o n v}$ as hysteresis develops. The surface size function $x_{0}$ at the non-loaded state and the function $x_{c o n v}$ after a number of inelastic cycles were adopted as the F.S.S. functions. $\varkappa_{0}$ and $\varkappa_{\text {conv }}$ were obtained by a tensile test and a tension-compression test, while the weighting function $W$ was determined through trial-and-error with numerical calculations.

The two quantities below were adopted in the model as state variables to express hysteretic effects.

$$
\bar{\varepsilon}_{p}=\int_{t_{0}}^{t_{c}} d \bar{\varepsilon}_{p}, \bar{\varepsilon}_{p i}=\int_{t_{c}}^{t_{i}} d \bar{\varepsilon}_{p}, d \bar{\varepsilon}_{p}=\sqrt{\frac{2}{3} d \varepsilon_{i j}^{p} d \varepsilon_{i j}^{p}}
$$

where, $\bar{\varepsilon}_{p}$ is cumulative equivalent plastic strain from the start time $\left(t_{0}\right)$ of loading to the time $\left(t_{c}\right)$ of the last unloading on a stress-strain path, and $\bar{\varepsilon}_{p i}$ is equivalent plastic strain increment from the time $\left(t_{c}\right)$ to

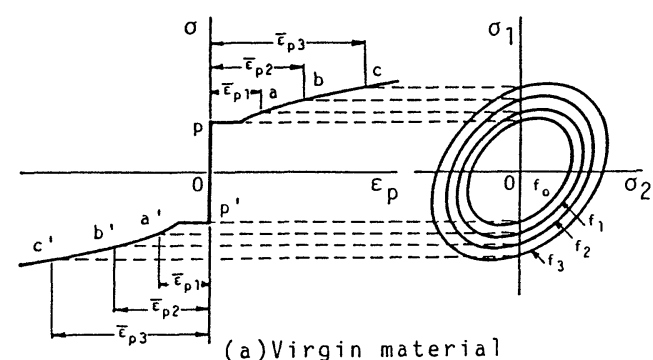

(a) Virgin material

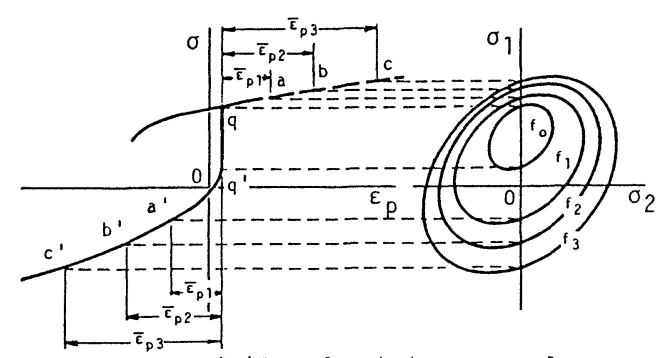

(b) Pre-loaded material

Fig. 1 Multi-surface plasticity model ${ }^{10), 11)}$.

* Although in the references 14) and 15), the authors used the notations of $x_{a}$ and $x_{b}$ following the paper presented by Petersson and Popov, we changed the subscripts to express appropriately the meanings of these functions as shown in this paper. 
the present time $\left(t_{i}\right) . \varkappa_{0}$ and $\varkappa_{\text {conv }}$ obtained as functions of $\bar{\varepsilon}_{p i}$, and $W$ which is a function of $\bar{\varepsilon}_{p}$ are employed to determine the surface size function $x$.

When the increase of the equivalent plastic strain occurs, vector $\{\alpha\}$ expressing the coordinates of the centers of the surfaces is successively updated as

$$
\left\{\alpha_{i}\right\}=\left\{\tilde{\alpha}_{j}\right\}+\frac{\{\tilde{\sigma}\}-\left\{\tilde{\alpha}_{0}\right\}}{\tilde{x}_{0}}\left(\tilde{x}_{j}-x_{i}\right)
$$

The subscripts $i$ and $j$ indicate the surfaces corresponding to the equivalent plastic strain increments being a certain value $\bar{\varepsilon}_{p i}$ and $\bar{\varepsilon}_{p i}+d \bar{\varepsilon}_{p}$, respectively. For example, letting $\bar{\varepsilon}_{p 2}=\bar{\varepsilon}_{p i}$ and $\bar{\varepsilon}_{p 3}=\bar{\varepsilon}_{p i}+d \bar{\varepsilon}_{p}$ in Fig. 1, the surfaces $f_{2}$ and $f_{3}$ become surface $i$ and surface $j$, respectively. The $\{\sigma\}$ indicates stress vector, and the subscript ${ }_{0}$ indicates $\bar{\varepsilon}_{p i}=0$. Further, indicates before updating.

\section{PROPOSED MODEL ${ }^{14), 15)}$}

The stress-strain model proposed was constructed based on experimental results obtained by the authors, with an intention to make a model that could accurately pursue the results of measurements. Principal features are described below.

\section{(1) Evaluation of cumulative equivalent plastic strain}

The accumulation method of the equivalent plastic strain during repetitive loading is consistent with the experimintal results obtaind by the authors ${ }^{15)}$ and the return phenomenon presented by Yokoo et al. ${ }^{16)}$. As shown in Fig. 2 (a), of the equivalent plastic strain occurring on a certain path, only the part exceeding the maximum equivalent plastic strain having been occurred up to that time is considered to affect the subsequent hysteresis, and is accumulated to make up the cumulative equivalent plastic strain. On the contrary, cumulative equivalent plastic strain of Petersson-Popov Model is calculated by integration of the equivalent plastic strain on the entire strain paths as shown in Fig. 2(b).

(2) Fundamental surface size functions and weighting functions

Ordinarily, in case a steel specimen is stretched and unloading occurs before the yield plateau ends, a yield phenomenon appears in the reversed loading region. When unloading occurs immediately before strain hardening starts after the yield plateau has ended, the yield phenomenon no longer appears, and there is a gradual stress-strain relation in which the Bauschinger effect appears prominently. Further, this phenomenon is similarly found in case also of unloading occurring at the strain hardening region, but the degree of the Bauschinger effect is not the same. Once the hysteresis has progressed to the strain-hardening region, the yield plateau no longer appears and the degree of the Bauschinger effect changes notably along with the progress of hysteresis. In order to express such a property of the steel, following three states were selected as the fundamental states, namely, the state with no hysteretic effect at all, the state when the yield plateau ends, and the state when the Bauschinger effect converges (i. e., the hysteresis becomes stationary ). $\varkappa_{0}, \varkappa_{s t}$, and $\varkappa_{\text {conv }}$ defined as the surface sizes at those states were adopted as F.S. S. functions.

Whereas the three functions of $\varkappa_{0}, x_{s t}$, and $\varkappa_{c o n v}$ were selected as F.S.S. functions, two weighting functions, $W_{1}$ and $W_{2}$ were defined. The functions $W_{1}$ is one in order to express the transition from the virgin stress-strain curve having a yield plateau and strain hardening region to a gradual curve, while the function $W_{2}$ is one for expressing the change in the degree of the Bauschinger effect. The function $x$ at a certain hysteresis stage is defined by the following equation.

$$
\varkappa= \begin{cases}W_{1} \varkappa_{0}+\left(1-W_{1}\right) \varkappa_{s t} & ; 0 \leqq \bar{\varepsilon}_{p}<\bar{\varepsilon}_{p, s t} \\ W_{2} \varkappa_{s t}+\left(1-W_{2}\right) \varkappa_{c o n v} & ; \bar{\varepsilon}_{p, s t} \leqq \bar{\varepsilon}_{p}<\bar{\varepsilon}_{p, \text { conv }} \\ \varkappa_{\text {conv }} & ; \bar{\varepsilon}_{p, \text { conv }} \leqq \bar{\varepsilon}_{p}\end{cases}
$$

where $\bar{\varepsilon}_{p, s t}$ is the cumulative equivalent plastic strain when the yield plateau ends and $\bar{\varepsilon}_{p, \text { conv }}$ is that when the hysteresis becomes stationary. In this equation, $W_{1}=1$, when $\bar{\varepsilon}_{p}=0, W_{1}=0$ and $W_{2}=1$ when $\bar{\varepsilon}_{p}=\bar{\varepsilon}_{p, s t}$, and $W_{2}=0$ when $\bar{\varepsilon}_{p}=\bar{\varepsilon}_{p, \text { conv }}$. 
In order to use the proposed model, simple experiments are to be performed and the weighting functions should be determined by means of these experimental results. According to the experimental results obtained by the authors, there was a trend that the weighting functions varied in relation to $\bar{\varepsilon}_{p i}$. Therefore, from the standpoint that modelling should be faithful to the experimental results, the weighting functions were formulated as $W=W\left(\bar{\varepsilon}_{p}, \bar{\varepsilon}_{p i}\right)$.

(3) Method for evaluating material parameters

Since the proposed model is based on the hypothesis that the sizes of the loading surfaces are determined by the cumulative equivalent plastic strain $\bar{\varepsilon}_{p}$ only up to that time, it is possible to evaluate the material parameters by means of stress-strain relations obtained by monotonous loading tests and tensioncompression loading tests including load reversal occurring just once.

\section{INFLUENCES OF VARIOUS FACTORS ON ACCURACIES OF PREDICTED HYSTERETIC STRESS-STRAIN RELATIONS}

In case of predicting hysteretic stress-strain relations of steel by means of the proposed model, calculated results depend on a number of factors, and these factors have serious effects on prediction accuracies. Thereupon, these factors are extracted to quantitatively grasp the effects on the accuracies, and effectiveness of the model is examined.

Here examinations are made of the effects of the three factors below thought to have especially substantial effects on prediction accuracies.

a) Method for evaluating cumulative equivalent plastic strain

b) Method for selecting F. S. S. functions

c) Configuration of weighting functions

Focused on these factors, eight models listed in Table 1 are set up.

Regarding Factor a), two methods for the accumulation on the entire strain paths and on the partial paths as mentioned in Section 3. (1) are considered (see Fig. 2).

With regard to Factor b), as shown in Fig. 3, classifications are made for cases of using only $\chi_{0}$ and $\varkappa_{c o n v}$, and those of additionally introducing $\varkappa_{s t}$. In the former, only $W$ is defined as a weighting function, while in the latter, two weighting functions $W_{1}$ and $W_{2}$ are used as mentioned in Section 3 . (3).

Concerning Factor c), five functions shown in Fig. 3 are used. With regard to the case in which $\chi_{s t}$ is not introduced as one of the F.S.S. functions, a function decreasing sharply as shown in Ref. 10), and a function which decreases linearly in relation to $\bar{\varepsilon}_{p}$ are set up. In case of introducing $\varkappa_{s t}$, three types of functions are employed; a function $W=W\left(\bar{\varepsilon}_{p}, \bar{\varepsilon}_{p i}\right)$ mentioned in Section 3. (3), a function $W=W\left(\bar{\varepsilon}_{p}\right)$ constant in relation to $\bar{\varepsilon}_{p i}$, and a function constant in relation to $\bar{\varepsilon}_{p i}$ and decreasing linearly in relation to $\bar{\varepsilon}_{p}$.

Table 1 Definitions of models.

\begin{tabular}{|c|c|c|c|}
\hline name & accumulation of $\bar{\varepsilon}_{p}$ & $\begin{array}{c}\text { fundamental surface } \\
\text { size functions }\end{array}$ & weighting function \\
\hline$A-2-P^{* 2}$ & over all paths & $\kappa_{0}$ and $\kappa_{\text {conv }}$ & by $\operatorname{Ref.}[10]$ \\
\hline$A-2-L$ & over all paths & $\kappa_{0}$ and $\kappa_{\text {conv }}$ & linear \\
\hline$P-2-P$ & as shown in Fig.2(a) & $\kappa_{0}$ and $\kappa_{\text {conv }}$ & by $\operatorname{Ref} .[10]$ \\
\hline$P-2-L$ & as shown in Fig.2(a) & $\kappa_{0}$ and $\kappa_{\text {conv }}$ & linear \\
\hline$A-3-M$ & over all paths & $\kappa_{0}, k_{s t}$ and $\kappa_{\text {conv }}$ & measured ${ }^{* 3}$ \\
\hline$P-3-M$ & as shown in Fig.2(a) & $\kappa_{o}, \kappa_{s t}$ and $\kappa_{\text {conv }}$ & measured ${ }^{* 3}$ \\
\hline$P-3-M 2^{* 1}$ & as shown in Fig.2(a) & $\kappa_{0}, \kappa_{s t}$ and $\kappa_{\text {conv }}$ & measured ${ }^{* 4}$ \\
\hline$P-3-L$ & as shown in Fig.2(a) & $\kappa_{0}, \kappa_{s t}$ and $\kappa_{\text {conv }}$ & linear \\
\hline
\end{tabular}

*1 This model is proposed by the authors ${ }^{15}$ ).

*2 This model is proposed by Petersson and Popovio),11).

*3 In these models only $\bar{\varepsilon}_{\mathrm{p}}$ is a variable.

*4 In this model $\bar{\varepsilon}_{\mathrm{p}}$ and $\bar{\varepsilon}_{\mathrm{p} \mathrm{i}}$ are variables. 


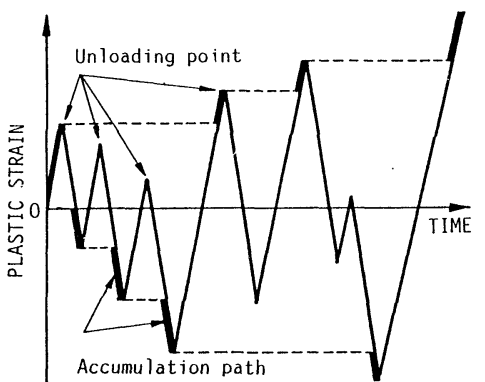

(a) method no.1

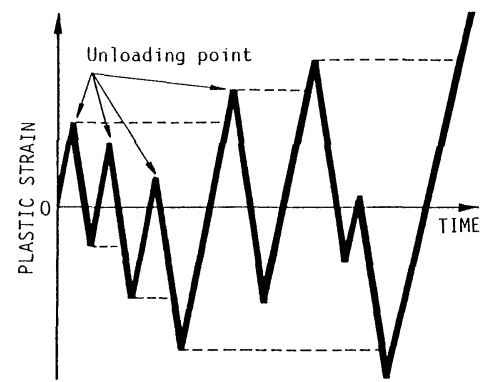

(b) method no.2

Fig. 2 Accumulation methods of plastic strain.
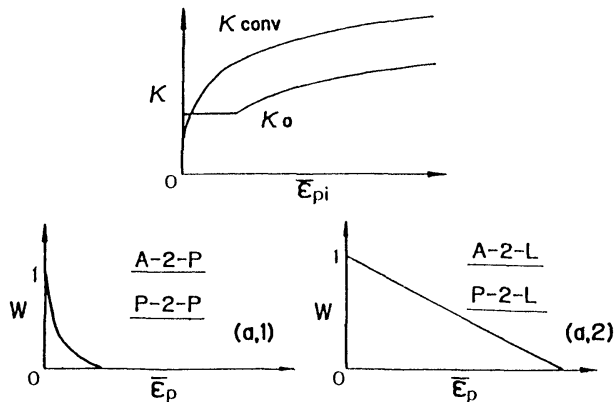

(a) Case of two fundamental surface size function

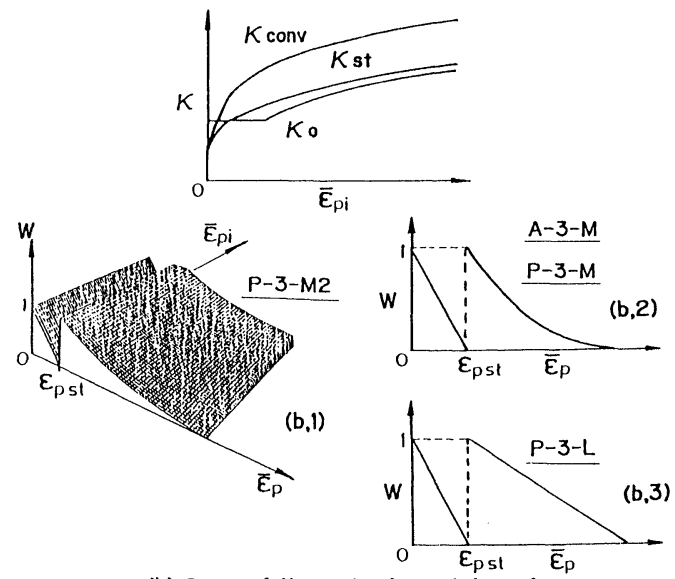

(b) Case of three fundamental surface size function

Fig. 3 Fundamental surface size functions and weighting functions for each models.

F.E.M. analyses ${ }^{17) .18)}$ were performed by means of the cyclic plasticity models shown in Table 1 for round-bar specimens subjected to repetitive tension-compression loads by controlled strain. The calculated results and the results obtained by corresponding experiments carried out by the authors ${ }^{15)}$ are compared.

In order to examine accuracies of the stress-strain relations, the value $\gamma$ given by the following equation is defined as an error measure.

$$
\gamma=\frac{\int_{\text {all paths }}\left|\sigma_{\text {i.cal. }}-\sigma_{\text {i.exp. }}\right| d \varepsilon_{i}}{\int_{\text {all paths }}\left|\sigma_{\text {i.exp. }}\right| d \varepsilon_{i}} \times 100(\%)
$$

where $\sigma_{i}$ and $\varepsilon_{i}$ are stress and strain increment, respectively, from the $i$-th reverse point. Fig. 4 shows conceptually a meaning of the value $\gamma$. In order to calculate the quantity, calculated stress-strain curves of each strain paths are put upon curves measured, making start points of these curves coincide. The area bounded by these curves is denoted as "B", while the area " $\mathrm{A}$ " corresponds to strain energy accumulated on the path. The value $\gamma$ is the quantity obtained by the summation of the ratio $(\mathrm{B}) /(\mathrm{A})$, indicated by percentages.

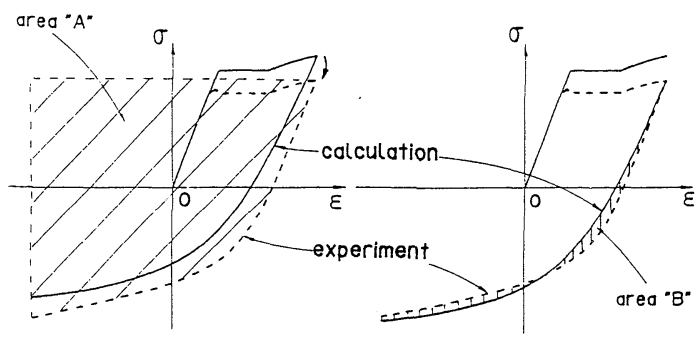

Fig. 4 Definition of error measure. 
Figs. 5 to 10 show comparisons of stress-strain relations obtained by each models focusing on the three factors mentioned above and on the $\gamma$ values. In these figures, (a) shows an example in case that a specimen is subjected to random strain cycles, and ( $b$ ) is one by which the effect of the method for the evaluation of the cumulative equivalent plastic strain is clearly indicated.

In Table 1, Model A-2-P corresponds to the model presented by Petersson and Popov, while Model $\mathrm{P}-3-\mathrm{M} 2$ is the model proposed by the authors (that is the proposed model). Therefore, as the names of these models imply, the evaluation method of the cumulative equivalent plastic strain in the Proposed Model as well as the number of the F.S.S. functions and evaluation method of the weighting functions differ from those in the Petersson-Popov Model. Therefore, it is impossible to clarify the influences of Factors a) to c), which have been listed in this section, on prediction accuracies by direct comparisons of stress-strain relations calculated by the proposed model and those predicted by the Petersson-Popov Model. Beginning in the following section, the influences of those factors on accuracies of calculated stress-strain relations are individualy investigated.

\section{INFLUENCE OF METHOD FOR EVALUATING CUMULATIVE EQUIVALENT PLAS- TIC STRAIN}

When $x_{s t}$ is not introduced as one of the F.S. S. functions, variation in the surface size function from $x_{0}$ to $x_{\text {conv }}$ depends on the degree of increase in the cumulative equivalent plastic strain due to repetitions of plastic strain, and the degree of reduction of weighting function in relation to the increasing cumulative equivalent plastic strain.

In case the weighting function is sharply decreased, the difference due to the method of evaluation of the cumulative equivalent plastc strain, if the range of small strains is excepted, will not be large compared with that in case of the weighting function being gradually decreased. Figs. 5 (a) and (b) compare the results of calculations with Models A-2-P and P-2-P. In the case of (a) there is hardly any difference. In the case of (b) also, the difference is not large if stress amplitudes are focused on. Further, from the fact
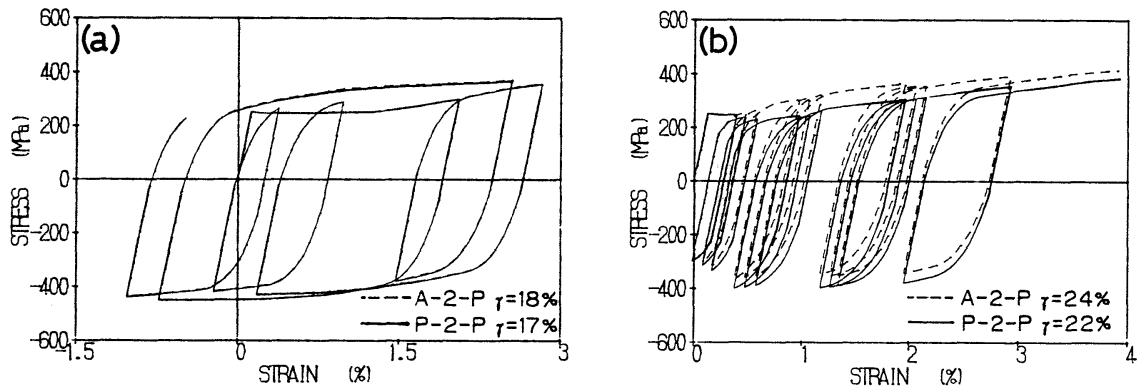

Fig. 5 Influence of the method for evaluating $\bar{\varepsilon}_{p}$ in case the weighting function decreases sharply ; Comparisons of Models A-2-P and $\mathrm{P}-2-\mathrm{P}$.
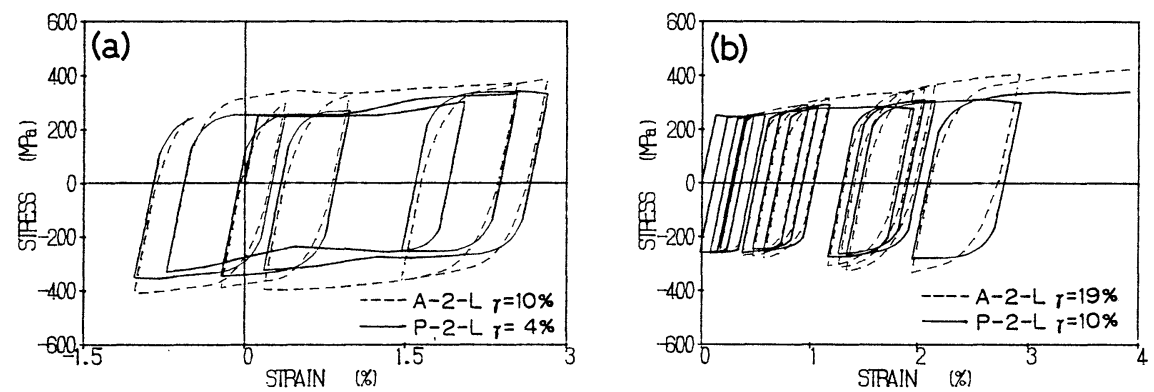

Fig. 6 Influence of the method for evaluating $\bar{\varepsilon}_{\rho}$ in case the weighting function decreases gradually ; Comparisons of Models A-2-L and P-2-L. 
that the weighting function is sharply reduced, the yield plateau disappears from the second path in the case of (a).

When the weighting function is gradually decreased, the difference between Model A-2-L and Model P-2-L is shown in Figs. 6 (a) and (b). The results are remarkably different depending on the method for evaluating the cumulative equivalent plastic strain. In contrast to the case of Model P-2-L, where the yield plateau appears due to the effect of $x_{0}$ until after the hysteresis has progressed to a fair extent, it disappears at a comparatively early time in Model A-2-L. However, compared with Model A-2-P and Model P-2-P, there is some effect remaining in the results with Model A-2-L.

In view of these facts, when $\chi_{s t}$ is not introducd, it is necessary to use the weighting function decreasing abruptly against the increase in the cumulative equivalent plastic strain in order to express the process of the yield plateau disappearing. In this case, the effect of $\varkappa_{0}$ on the stress-strain relation becomes small, but inversely, the effect of $x_{c o n v}$ becomes excessive. There are cases of the value of $\gamma$ being 10 to 30 percent, and accurate predictions have not been done.

According to the measurements by the authors, $\varkappa_{c o n v}$ is considerably larger than $\chi_{0}$ in the range of large strain, while these functions shown in Ref. 10) are identical in the range of large strain. In case $\varkappa_{\text {conv }}$ is notably larger than $\varkappa_{0}$, when the weighting function is sharply reduced, since there is such a rapid change in the surface size function from $x_{0}$ to $x_{\text {conv }}$, enlargement of the surface sizes will be overestimated. Consequently, although the configuration of the stress-strain curve will become gradual, the stress value will be calculated on the large side. In other words, when expressing stress-strain behaviours of a material which indicates yield plateau and notable strain hardening, it is necessary to use a weighting function which decreases rapidly for the process of disappearance of the yield plateau and to employ a weighting function which decreases gradually for the process of change in the Bauschinger effect. Thus the two processes require opposing weighting functions.

Thereupon, when the surface size function $\chi_{s t}$ at the time of ending of the special phinomenon called the yield plateau is introduced as one of the F. S. S. functions, and different weighting functions are used for the processes of $\varkappa_{0}$ to $\varkappa_{s t}$ and $\varkappa_{s t}$ to $\varkappa_{c o n v}$, it is expected that the model will be capable of expressing both of these two phenomena. Further, the authors obtained experimintal results that the weighting function for expressing the transition from $\varkappa_{s t}$ to $\varkappa_{c o n v}$ gradually decreased as the cumulative equivalent plastic strain increased. Therefore, it can be surmised from comparisons of Model A-2-L and Model P-2-L and Model P-2-L (see Fig. 6) that the method for evaluating the cumulative equivalent plastic strain will have considerable influences on prediction accuracies of stress-strain relations after ending of the yield plateau.

\section{INFLUENCE OF INTRODUCTION OF ADDITIONAL FUNDAMENTAL SURFACE SIZE FUNCTION}

In the foregoing section, it was indicated that in case that $\chi_{0}$ and $\chi_{c o n v}$ had fairly different values in the range of large strain such as the yield plateau was exceeded, it was necessary to additionally introduce $x_{s t}$ as one of the F.S. S. functions in order to express faithfully actual behaviours of steel. In this section, examinations will be made of how numerical calculation results will be improved in case of introducing $\varkappa_{s t}$.

Figs. 7 (a) and (b) show comparisons of Model P-2-L and Model P-3-M. Compared with the results with Model P-2-L in which the influence of the yield plateau is apparent even after considerable histories, stress-strain curves with Model P-3-M show predominant Bauschinger effect in the second path and after. Moreover, since the method for evaluating the cumulative equivalent plastic strain has been improved, the value of $\gamma$ is held within 5 percent at most, and enlargement of the surfae sizes is not overestimated.

Figs. 8 (a) and (b) compare the results of calculations with Model P-3-M and Model A-3-M. Although completely identical stress-strain relations are obtained up to the second path, on a subsequent path, difference of those relations appears due to the difference in the method for evaluating the cumulative 

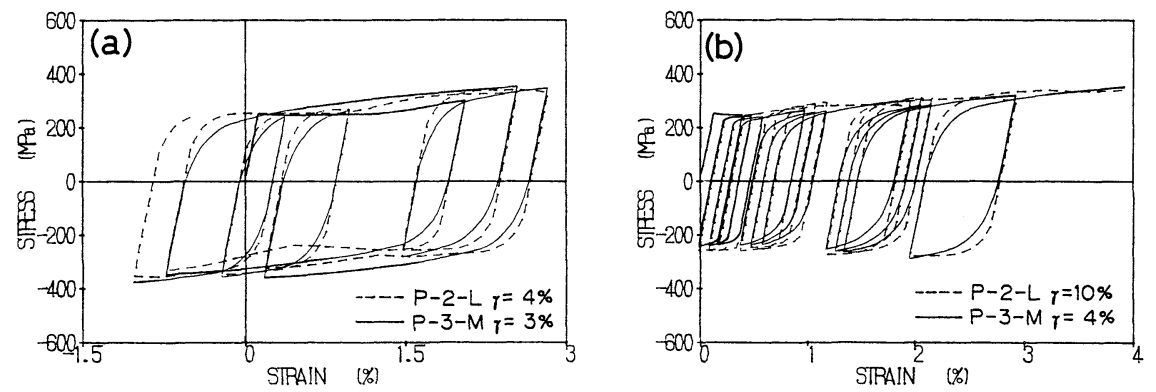

Fig. 7 Influence of introduction of additional F. S. S. function in case the weighting function decreases gradually ; Comparisons of Models P-2-L and P-3-M.


Fig. 8 Influence of the method for evaluating $\bar{\varepsilon}_{p}$ in case the function $x_{s t}$ is additionally introduced ; Comparisons of Models A-3-M and $\mathrm{P}-3-\mathrm{M}$.

equivalent plastic strain. In Model A-3-M, all of the equivalent plastic strain at the second path is accumulated to determine the surface size function at the third path. On the contrary, in Model P-3-M, only compression plastic strain is accumulated. Then, the surface sizes in Model P-3-M become smaller than those in Model A-3-M. Subsequently, this trend appears prominently in all of the cycles with the surface size function abruptly becoming $\chi_{\text {conv }}$ in Model A-3-M so that stress values become large in relation to measured values similarly to Model A-2-P, Model A-2-L, and Model P-2-P. As a result, the values of $\gamma$ in these examples are approximately 10 to 20 percent, and in case of using the value accumulated for the entire paths as the cumulative equivalent plastic strain, the accuracies of the stress-strain relations calculated are extremely impaired.

\section{INFLUENCE OF SIMPLIFICATION OF WEIGHTING FUNCTION}

In this section, an examination is made of the degree of influence that the simplification of the weighting function will have on calculated results when the abovementioned evaluation of the cumulative equivalent plastic strain and introduction of the surface size function $x_{s t}$ as one of the F. S. S. functions are done.

(1) Simplification of measured weighting function

Fig. 9 (a) and (b) compare the results of calculations with Model P-3-M2 and Model P-3-M. In the former, the weighting function is formulated as $W=W\left(\bar{\varepsilon}_{p}, \bar{\varepsilon}_{p i}\right)$, while it is defined as $W=W\left(\bar{\varepsilon}_{p}\right)$ in the latter. The differences in the stress-strain relations obtained by these models are extremely slight. According to these figures, the influence of the simplification of measured weighting function is negligible. If the accuracy is of approximately the same degree, it is desirable for the model to be simple as much as possible, and necessity is not recognized for a change to be made from Model P-3-M to $\mathrm{P}-3-\mathrm{M} 2$ with the material used here. However, this can be said for only the cases of the material used by the authors, and it will be necessary to adopt Model P-3-M2 for materials with higher ratios of change in the weighting function in relation to the cumulative equivalent plastic strain increment. 

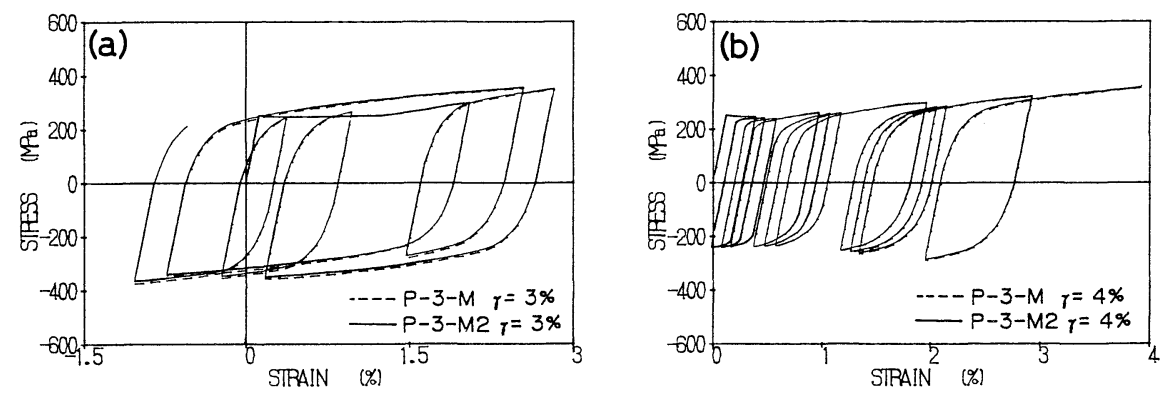

Fig. 9 Influence of simplification of weighting function ; Comparisons of Models $\mathrm{P}-3-\mathrm{M}$ and $\mathrm{P}-3-\mathrm{M} 2$.
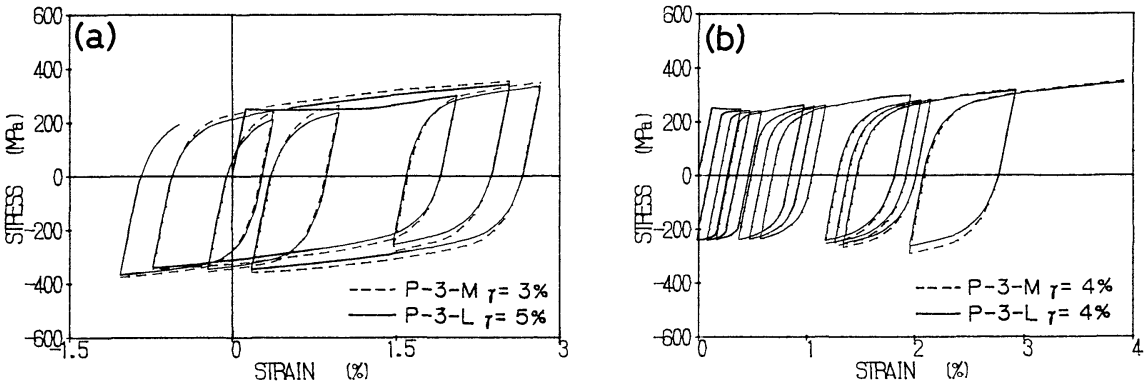

Fig. 10 Influence of linearlization of weighting function ; Comparisons of Models P-3-M and P-3-L.

\section{( 2 ) Linearlization of weighting function}

Carrying out monotonous tensile tests and tension-compression tests each including just one load reversal with several specimens and employing the method for evaluating material parameters proposed by the authors, it is possible to accurately estimate hysteretic stress-strain relations by Model P-3-M. However, if it were to be possible to assume that the weighting function decreases linearly as the cumulative equivalent plastic strain increases, only the experiments for determining the functions $x_{0}, x_{s t}$, and $\varkappa_{\text {conv }}$ are required. Then, comparisons are made of the results of numerical calculations with Model P-3-M by means of the weighting function obtained by the authors' measurements and those with Model P-3-L by a weighting function which decreases linearly as the cumulative equivalent plastic strain increases, and the results are shown in Figs. 10 (a) and (b).

In case of the measured weighting function protruding downward in relation to the cumulative equivalent plastic strain such as with the material used here ${ }^{15)}$ stress occurs on the slightly small side, but the degree of influence is considerably small when compared with the influences of Factors a) and b) cited in Section 4 .

\section{COMPARISONS OF PROPOSED MODEL AND PETERSSON-POPOV MODEL}

Figs. 11 (a) and (b) compare the calculated results with the proposed model (that is Model P-3-M2) and Petersson-Popov Model (that is Model A-2-P), while Figs. 12 (a) and (b) show stress-strain relations calculated with the proposed model and those gained by the corresponding experiments ${ }^{15}$. These figures show that the effects of Factors a) and b) cited in Section 4 are significant as previously investigated and the model proposed by the authors becomes excellent by the modifications mentioned in Section 3 compared with the original Petersson-Popov Model.

\section{CONCLUSIONS}

The conclusions obtained within the scope of this study are summarized as follows.

(1) Prediction accuracies of stress-strain relations are improved by employing the method, which is 

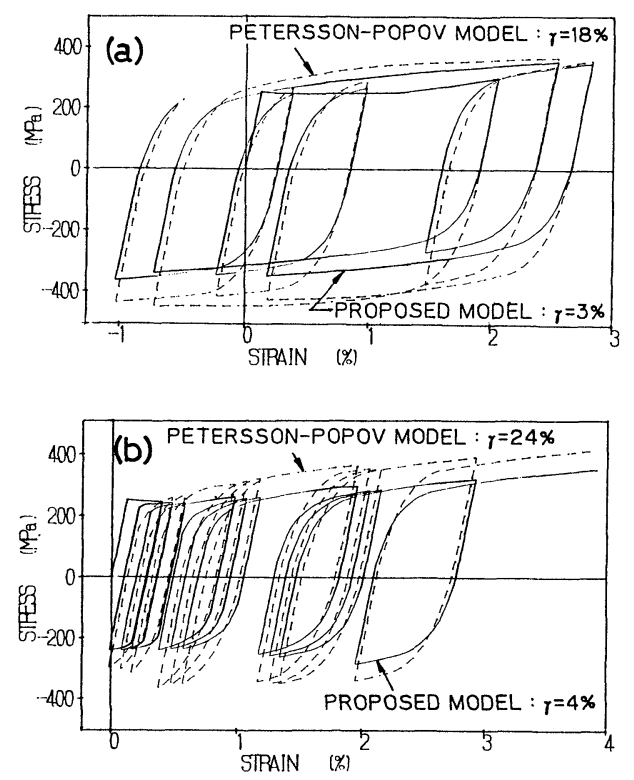

Fig. 11 Comparisons of stress-strain relations ; proposed model (“P-3-M2”) and Petersson-Popov Model (“A-2-P”).
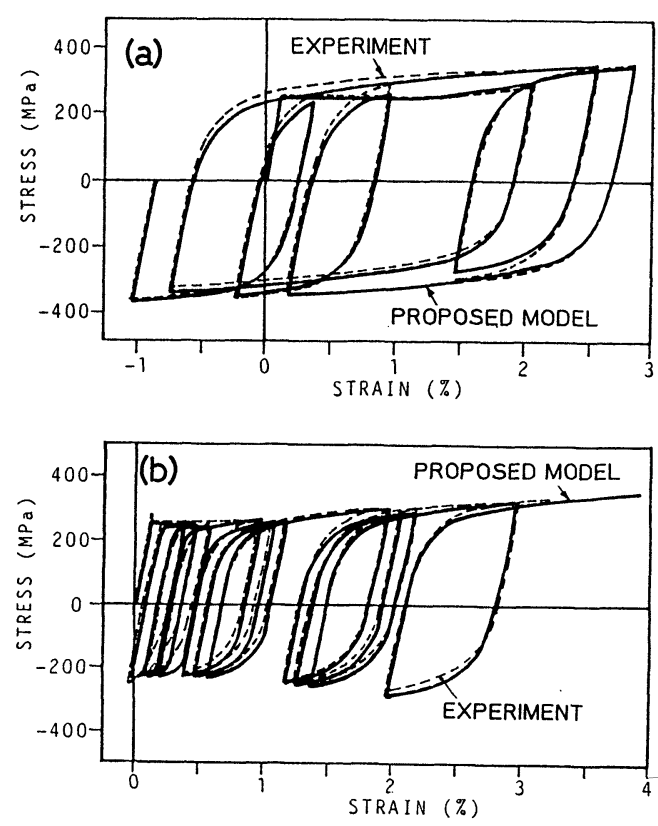

Fig. 12 Comparisons of stress-strain relations; experiments and calculations by the proposed model ${ }^{15}$.

shown in Fig. 2(a), for evaluating the cumlative equivalent plastic strain. The error in case of using the value accumulated for all of the paths is 10 to 30 percent at times in the examples of measurements reported herein.

(2) In case of a steel for which the yield plateau appears prominently, it becomes possible for a realistic estimation of stress-strain relations to be made through the introduction of the surface size function $\varkappa_{s t}$ at the time of ending of the yield plateau as one of the fundamental surface size functions.

(3) Even though the weighting function is assumed to be constant for a monotonous stress-strain path, the error due to this simplification can be ignored.

(4) In case of using the weighting function decreasing linearly in relation to the cumulative equivalent plastic strain, measurements need not be carried out in order to determine the weighting function and the error appearing in the stress-strain relation was about 5 percent in relation to the results of calculations with the weighting function obtained by measurements.

(5) The errors in the stress-strain relations calculated by means of the proposed model were about 5 percent at most in relation to experimental results.

\section{ACKNOWLEDGMENT}

The numerical calculations in this study were performed using a HITAC M-280 H/200 and M- $680 \mathrm{H}$ at the Computer Center, the University of Tokyo. A part of this study was sponsored by the Grant-in-Aid for Scientific Research of the Ministry of Education, Science and Culture.

\section{REFERENCES}

1) Hill, R. : The Mathematical Theory of Plasticity, Oxford at the Clarendon Press, 1950 ( translated into Japanese by Washizu, K., Yamada, Y. and Kudo, H., Baihukan, 1954).

2) Prager, W. : The theory of plasticity : A survey of recent achievements, Proc. of the Institute of Mechanical Engineers, Vol. 169, pp.41-57, 1955.

3) Ziegler, H. : A modification of Prager's hardening rule, Quarterly of Applied Mathematics, Vol. 17, pp. 55-65, 1959.

4) Duwez, P. : On the plasticity of crystals, Physical Review, Vol.47, pp.494-501, 1935. 
5) Mróz, M. : An attempt to describe the behavior of metals under cyclic loading using a more general workhardening model, Acta Mechanica, Vol. 7, pp. 199-212, 1969.

6) Dafalias, Y.F. and Popov, E. P. : A model of nonlinear hardening materials for complex loadings, Acta Mechainica, 21, pp. 173-192, 1975.

7) Krieg, R. D. : A practical two surface plasticity theory, Trans. ASME, Jour. Applied Mechanics, Vol. 42, pp. 641-646, 1975.

8) Dafalias, Y.F. and Popov, E. P. : Rate-independent cyclic plasticity in a plastic internal variables formalism, Mechanics Research Commumications, Vol.3, pp. 33-38, 1976.

9) Dafalias, Y. F. and Popov, E. P. : Plastic internal variables formalism of cyclic plasticity, Journal of Applied Mechanics, Paper No.76-WA/APM-21, American Society of Mechanical Engineers, Vol.43, pp.645-651, December 1976.

10) Petersson, H. and Popov, E. P. : Constitutive relation for generalized loadings, Proc. of ASCE, Vol. 103, No. EM 4, pp. 611-627, 1977.

11) Popov, E. P. and Petersson, H. : Cyclic metal plasticity; Experiments and theory, Proc. of ASCE, Vol. 104, No. EM6, pp. 1371-1388, 1978.

12) Fujimoto, M., Nakagome, T. and Yamada, T. : Mechanical study of steel structure connections subjected to cyclic force, Proc. of 15th Congress, Japan Steel Structures Council, “Study on Matrix Analysis Method," pp. 161-166, 1981 (in Japnese).

13) Cofie, N. G. and Krawinkler, H. : Uniaxial cyclic stress-strain behaviors of structural steel, Proc, of ASCE, Vol.111, No. EM9, pp. 1105-1120, 1985.

14) Minagawa, M., Nishiwaki, T., Masuda, N. and Saito, T. : High-accuracy stress-strain model of structural steel under cyclic loading, Proc. of the 9 th Symposium on Computational Methods in Structural Engineering and Related Fields, Japan Steel Structures Council, pp. 145-150, July 1985 (in Japanese).

15) Minagawa, M., Nishiwaki, T. and Masuda, N. : Modelling cyclic plasticity of structural steels, Structural Engineering/ Earthquake Engineering, Vol.4, No. 2, pp.361s-370s (Proc. of the Japan Society of Civil Engineers, No. 368/I-8, pp. 145-154), October 1987.

16) Yokoo, Y., Nakamura, T. and Kawada, Y. : Non-stationary hysteretic uniaxial stress-strain relation of a wide-flange steel (Part I), Trans. of A. I. J., No. 259, pp.55-66, 1977, and Yokoo, Y., Nakamura, T. : Non-stationary hysteretic uniaxial stress-strain relation of a wide-flangre steel (Part II), Trans. of A. I. J., No. 260, pp.71-81, 1977.

17) Yamada, Y. : Fundamentals and Applications of Finite Element Method Series, No. 6, Plasticity and Visco-elasticity, Baihukan Publishing Co., 1980 (in Japanese).

18) Yamada, Y. and Yokouchi, Y. : Programming of Elasto-plastic Analysis by the Finite Element Method-EPIC IV CommentaryBaihukan Publishing Co., 1981 (in Japanese).

(Received September 26 1988) 